\title{
Evidencias de validez de la escala para la Evaluación de la Vinculación Afectiva y la Adaptación Prenatal (EVAP) en gestantes usuarias del INMP
}

\section{Evidence of validity of the scale for the Evaluation of the Affective Bond and the Prenatal Adaptation (EVAP) in users of 1the INMP}

\author{
José Artica-Martínez ${ }^{1}$ \\ Gloria Barba-Aymar ${ }^{2}$ \\ Ana María Mejía-Muñoz ${ }^{3}$ \\ Ermes Manco-Ávila ${ }^{4}$ \\ Jimmy Orihuela-Salazar ${ }^{5}$ \\ Instituto Nacional Materno Perinatal
}

Recibido: $15-11-18$

Aceptado: $27-12-18$

\begin{abstract}
Resumen
La presente investigación de tipo instrumental tuvo como propósito desarrollar evidencias de validez y confiabilidad de la Escala para la evaluación de la vinculación afectiva y la adaptación prenatal (EVAP) en una población de gestantes del Instituto Nacional Materno Perinatal. Participaron de la investigación gestantes, adolescentes y adultas $(\mathrm{N}=310)$, a partir del segundo trimestre de embarazo; es decir, a partir de las 14 semanas. Los hallazgos del estudio concluyeron que las seis dimensiones propuesta por Lafuente representan microdimensiones de los constructos adaptación y vinculación afectiva prenatal; por tanto, no se requiere la subdivisión, siendo necesaria una estructura solo con dos dimensiones. La versión final de la escala quedó conformada por 21 ítems, doce integraron la dimensión Adaptación al embarazo y nueve conformaron la dimensión Vinculación afectiva prenatal. El análisis factorial confirmatorio demostró que esta versión de la escala presenta mejores bondades de ajuste en comparación con la propuesta teórica y la estructura sugerida por el análisis factorial exploratorio sin omisión de ítems. Por último, en cuanto a la confiabilidad de la escala se obtuvieron valores de .746 y .749 para las dimensiones Adaptación al embarazo y Vinculación afectiva prenatal respectivamente. Estos valores fueron estimados a través del coeficiente omega resultando adecuados para ambas subescalas.
\end{abstract}

Palabras clave: Adaptación; embarazo; afectividad prenatal; validez interna; confiabilidad.

\footnotetext{
1 Psicólogo del Instituto Nacional Materno Perinatal. Autor para correspondencia.

E-mail: juan.artica@unmsm.edu.pe

2 Psicóloga del Instituto Nacional Materno Perinatal. E-mail: gbarbaaymar@gmail.com

3 Jefa del servicio de psicología del Instituto Nacional Materno Perinatal. E-mail: amejia@iemp.gob.pe

4 Psicóloga del Instituto Nacional Materno Perinatal. E-mail: katymancoavila@hotmail.com

5 Psicólogo del Instituto Nacional Materno Perinatal. E-mail: jimmyorihuela@hotmail.com

(C) Los autores. Este artículo es publicado por la Revista de Investigación en Psicología de la Facultad de Psicología, Universidad Nacional Mayor de San Marcos. Este es un artículo de acceso abierto, distribuido bajo los términos de la licencia Creative Commons Atribucion - No Comercia_Compartir Igual 4.0 Internacional. (http://creativecommons.org/licenses/by-nc-sa/4.0/) que permite el uso no comercial, distribución y reproducción en cualquier medio, siempre que la obra original sea debidamente citada.
} 


\begin{abstract}
The purpose of this instrumental research was to develop evidence of duration and reliability for the evaluation of affective attachment and prenatal adaptation (EVAP) in a pregnant population of the National Maternal Perinatal Institute. Participation of pregnant, adolescent and adult research $(\mathrm{N}=310)$, from the second trimester of pregnancy; That is, starting at 14 weeks. The findings of the study concluded that the six proposed dimensions for the representation of microdimensions of the constructs of adaptation and prenatal affective bonding; Therefore, its subdivision is not required, but a single structure with two dimensions is needed. The final version of the scale was made up of 21 items, twelve integrated the Adaptation to pregnancy dimension and nine formed the dimension Prenatal affective bonding. The confirmatory factor analysis showed that this version of the escalation presents the best adjustment benefits compared to the theoretical proposal and the structure suggested by the exploratory factor analysis without omission of items. Finally, in terms of the reliability of the scale, values of .746 and .749 were obtained for the Adaptation to pregnancy and Affective Linking dimensions, respectively. These values were estimated through the omega coefficient that is suitable for the subscales.
\end{abstract}

Keywords: Adaptation; pregnancy; prenatal affectivity; internal validity; reliability.

\title{
INTRODUCCIÓN
}

El embarazo es un proceso con implicaciones biológicas, psicológicas y sociales no solo para la gestante sino también para su pareja y el bebé por nacer; por tanto, es necesario el cuidado de todos aquellos factores que intervienen en el proceso de adaptación y vinculación prenatal. La inclusión de los aspectos psicosociales en esta etapa intrauterina es relevante, cabe mencionar la aceptación al embarazo, la identificación con el rol materno, la calidad de la relación con la pareja, la calidad de la relación la madre, la preparación para el parto, el miedo y pérdida de control; además, de la preocupación por su propio bienestar y de su bebé (Lederman \& Weis, 2009).

Durante el embarazo, la gestante vivencia distintas emociones como la alegría la sorpresa, la realización, al igual que estrés, ansiedad y miedo (Rodrigues, et al, 1999); no obstante, es indispensable, su adaptación a su embarazo a fin de desarrollar una vinculación afectiva con su nonato; además, de identificarse con su rol materno (Hidalgo \& Menéndez, 2003).

En tal sentido, se requiere de un instrumento que mida tanto la adaptación al embarazo como el nivel de vinculación afectiva prenatal, encontrándose en el contexto de instrumentos de evaluación psicológica la Escala para la evaluación de la vinculación afectiva y la adaptación prenatal (EVAP) diseñada por $\mathrm{M}^{\mathrm{a}}$ Lafuente (Lafuente, 2008). Para la construcción de la escala la autora consideró los aportes de Lederman (Lederman \& Weis, 2009) y Hughes (Hughes, 1987) en cuanto a las siete dimensiones de adaptación psicosocial al embarazo y las áreas de evaluación parento-fetal-vinculación y diferenciación respectivamente.

Reconociendo el valor de este instrumento, el presente trabajo de investigación tiene como propósito desarrollar evidencias de validez de dicha escala en la población de gestantes del Instituto Nacional Materno Perinatal la cual complementará la 
entrevista psicológica perinatal aportando información respecto a las actitudes y comportamiento de la futura madre midiendo el nivel de adaptación y vinculación afectiva conjuntamente. Para Hidalgo \& Menéndez (2003) es requisito la adaptación al embarazo para el desarrollo de la vinculación afectiva.

La propuesta teórica de Lafuente (2008) para evaluar la adaptación y el vínculo afectivo prenatal está compuesta por seis factores los cuales abordan aspectos vinculados con la capacidad de diferenciación madre- feto, apoyo familiar, capacidad imaginativa, aceptación del embarazo, experiencias tempranas de la infancia y la calidad de la relación con su pareja.

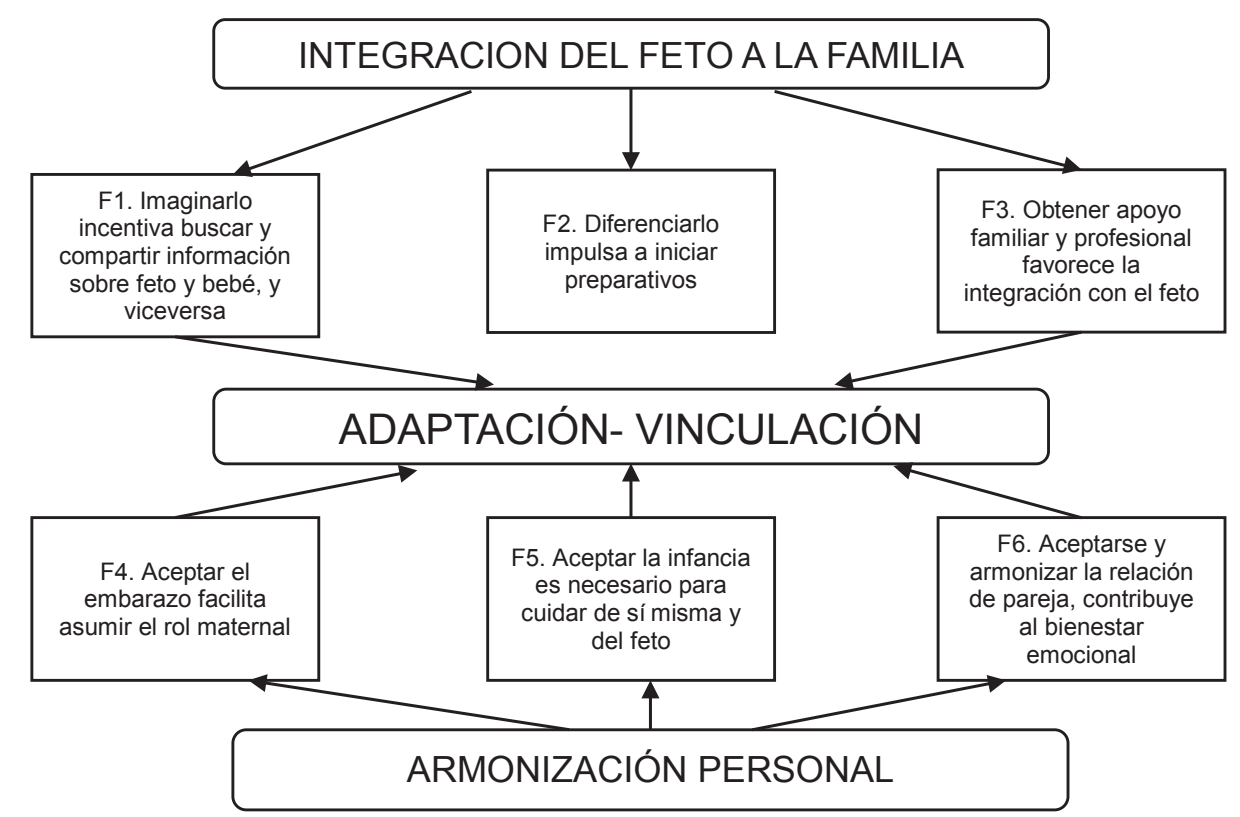

Figura 1. Estructura de la EVAP propuesto por Lafuente (2008)

La evaluación de todos estos aspectos permitirá identificar tempranamente aquellas conductas y/o sucesos psicosociales que estarían contribuyendo tanto positiva, como negativamente a este proceso. Con mayor énfasis en aquellos comportamientos que denoten una actitud de rechazo o indiferencia de la madre hacia su futuro hijo. Intervenir tempranamente mejoraría la calidad de la adaptación y vinculación afectiva prenatal.

La realización de esta investigación se justifica debido al interés de evaluar el modelo teórico de seis factores, que miden la adaptación y vinculación afectiva prenatal, propuesto por la autora de la escala; asimismo, reportar evidencias de validez y confiabilidad en una muestra peruana de gestantes. Igualmente, contar con un instrumento validado en nuestro contexto permitirá; en primer lugar, 
realizar estudios relacionando este constructo con otras con variables afines o sucesos significativos (life event) que podrían influir en el embarazo (Oiberman \& Galíndez, 2005) en segundo lugar, identificar tanto los recursos psicológicos de la gestante como aquellos comportamientos que indiquen rechazo o indiferencia hacia nonato a fin intervenir tempranamente y contribuir favorablemente al proceso de adaptación- vinculación prenatal. Por tanto, el objetivo de la presente investigación es desarrollar evidencias de validez y confiabilidad de la Escala para la evaluación de la vinculación afectiva y la adaptación prenatal en una población de gestantes del Instituto Nacional Materno Perinatal.

\section{MÉTODO}

La presente investigación corresponde a un estudio de tipo instrumental (Montero y León, 2002; Ato, López y Benavente, 2013) debido a que su propósito es analizar las propiedades psicométricas de la escala y determinar la calidad de su medición.

La población en estudio estuvo conformada por gestantes, adolescente y adulta, a partir del segundo trimestre de embarazo; es decir, a partir de las 14 semanas. En cuanto a la muestra participaron 310 gestantes usuarias del Instituto Nacional Materno perinatal.

En cuanto a los criterios de selección de las participantes, se consideraron gestantes adolescentes y adultas a partir del segundo trimestre del embarazo quienes consintieron su participación en el estudio firmando la ficha de consentimiento informado. Respecto a los criterios de exclusión solo se consideró no presentar alteraciones psicopatológicas.

De acuerdo con las sugerencias respecto a la adecuación del tamaño muestral en los estudios instrumentales (Comrey y Lee, 1992), se conformó la muestra con más de 200 participantes cantidad que es considerada como buena $(\mathrm{N}=310)$ a fin de obtener estimaciones precisas y estables.

Se inició la investigación solicitando la autorización de la autora de la escala en estudio, una vez obtenido ello, se diseñó el proyecto de investigación a fin de ser evaluado por la Unidad de Docencia e Investigación del INMP y facilitar su ejecución en la institución. La evaluación comprendió la revisión por el comité de metodología y ética.

Posteriormente, se continuó con el proceso de validación del instrumento siguiendo los estándares propuestos por la literatura (AERA, APA, \& NCME, 2014) se desarrolló evidencias de validez basadas en el contenido y en la estructura interna (Sireci y Padilla, 2014). Asimismo, se estimó la confiabilidad de la escala.

Por último, se elaboraron los baremos correspondientes a las variables la adaptación al embarazo y vinculación afectiva prenatal. 
Respecto al proceso de validación, se inició desarrollando evidencias de validez basada en el contenido; considerando este análisis necesario para la interpretación de las puntuaciones de la escala (Kane, 2009). Se realizó el análisis a través del Método basado en el juicio de expertos (Pedrosa, Suárez-Álvarez \& García-Cueto, 2013) estimando el índice de validez de contenido a través de valor de V de Aiken (Aiken, 1985). y sus Intervalos de confianza. Estos cálculos se realizaron con el programa informático en el lenguaje Visual Basic elaborado por Merino y Segovia (Merino y Segovia, 2009).

El análisis de la estructura interna del instrumento se evaluó mediante la técnica estadística del análisis factorial la cual consiste en identificar el número de factores subyacentes a los ítems; además, la pertenencia de cada ítem a un factor específico y el peso de cada ítem (Ventura-León, 2018) Con este propósito se realizó el análisis factorial exploratorio (AFE) y confirmatorio (AFC) para estimar la estructura de la escala. En cuanto al primer análisis, el AFE se realizó a través del programa Factor versión 10.8.04 (Lorenzo-Seva \& Ferrando, 2007) el cual brindó una propuesta tentativa sobre la estructura de la escala; en segundo lugar, se realizó un análisis confirmatorio (AFC) utilizando el programa AMOS en su versión 22 el cual permitió contrastar el modelo original de la autora con aquellos dos modelos propuestos a partir del análisis factorial exploratorio.

Por último, se estimó la confiabilidad a través del coeficiente omega el cual a diferencia de otros coeficientes trabaja con las cargas factoriales (Gerbing \& Anderson, 1988) lo cual permite obtener cálculos más estables reflejando el verdadero nivel de fiabilidad (Timmerman, 2005).

\section{RESULTADOS}

A continuación, se presentarán los resultados del proceso de validación de la escala tanto de contenido como de su estructura interna.

La tabla 1 muestra las puntuaciones obtenidas en los dos procesos de consulta a los jueces expertos respecto a la relevancia de los ítems para cada una de las seis dimensiones propuestas en el modelo teórico de la escala. Participaron ocho jueces expertos en el proceso de validación de contenido; además, de la relevancia se solicitó su apreciación sobre la claridad del ítem a fin de reformular su redacción.

Fue necesario realizar la evaluación en dos ocasiones debido a que la mayoría de los jueces presentaron observaciones a los ítems en la primera evaluación; además, que las puntuaciones obtenidas tanto en coeficiente V de Aiken (Aiken, 1996) como en el límite inferior de su Intervalo de confianza no fueron adecuados. Al respecto, dieciocho ítems no superaron el valor .70 en el límite inferior del IC al 95\%; puntuación mínima más exigente de validez (Charter, 2003). 
Tabla 1

Validez de contenido de la EVAP

\begin{tabular}{|c|c|c|c|c|}
\hline \multirow[b]{2}{*}{ Ítems } & \multicolumn{2}{|c|}{$\begin{array}{c}\text { Primera } \\
\text { aplicación }\end{array}$} & \multicolumn{2}{|c|}{$\begin{array}{c}\text { Segunda } \\
\text { aplicación }\end{array}$} \\
\hline & $\mathbf{V}$ & IC al $95 \%$ & $\mathbf{V}$ & IC al $95 \%$ \\
\hline 1. Imagino cómo es ahora mi hijo & .725 & $.553-849$ & .875 & $.719-.950$ \\
\hline 2. Imagino cómo será mi bebé & .875 & $.719-.950$ & .95 & $.816-988$ \\
\hline 3. Puedo hacerme una cierta idea del carácter de mi hijo & .725 & $.553-849$ & .825 & 672-.916 \\
\hline 4. He recogido información sobre el feto, sobre el bebé o sobre ambos & .85 & $.690-.935$ & .875 & $.719-950$ \\
\hline 5. Mi pareja y yo hablamos del futuro bebé & .875 & $.719-.950$ & .95 & $.826-.987$ \\
\hline 6. He pensado en la alimentación de mi futuro bebé & .65 & $.477-.791$ & .775 & $.606-885$ \\
\hline $\begin{array}{l}\text { 7. Cuando tuve mi primera falta (se me cayó el mundo encima... me llevé } \\
\text { una gran alegría...) }\end{array}$ & .7 & $.527-.830$ & .85 & $.7-.932$ \\
\hline $\begin{array}{l}\text { 8. Cuando pienso en mi vida con el bebé (me siento mal... tengo dudas... } \\
\text { creo que le gustaré y lo haré bien...) }\end{array}$ & .825 & $.672-.916$ & .9 & $.750-.964$ \\
\hline 9. Cuando se confirmó mi embarazo (no lo deseaba... lo acepté bien...) & .775 & $.606-885$ & .775 & $.616-.881$ \\
\hline 10. Creo que el embarazo (es un auténtico fastidio... es algo muy especial) & .75 & $.579-.867$ & .9 & $.750-.964$ \\
\hline $\begin{array}{l}\text { 11. Me resulta fácil imaginarme a mí misma dando de comer al bebé, } \\
\text { cuidándolo... }\end{array}$ & .825 & $.661-.919$ & .825 & $.661-.919$ \\
\hline 12. Siempre me han gustado los niños. & .825 & $.661-.919$ & .7 & $.527-.830$ \\
\hline $\begin{array}{l}\text { 13. Cuando hablo de mi futuro hijo suelo llamarle (esa cosa... el feto... mi } \\
\text { hijo, mi niño) }\end{array}$ & .775 & $.606-.885$ & .9 & $.750-.964$ \\
\hline 14. He elegido un nombre para mi hijo & .875 & $.719-.950$ & .9 & $.750-.964$ \\
\hline $\begin{array}{l}\text { 15. Cuando mi hijo se mueve (me molesta... me resulta indiferente... me } \\
\text { gusta) }\end{array}$ & .9 & $.750-.964$ & .95 & $.816-.988$ \\
\hline 16. He empezado a preparar algunas cosas para el nacimiento del bebé. & .9 & $.750-.964$ & .85 & $.690-.935$ \\
\hline 17. He pensado en la vuelta a casa desde el hospital & .725 & $.553-.849$ & .775 & $.606-.885$ \\
\hline $\begin{array}{l}\text { 18. El embarazo me ha producido (muchas molestias... algunas... ha sido } \\
\text { excelente) }\end{array}$ & .825 & $.661-.919$ & .775 & $.606-.885$ \\
\hline 19. Evito realizar actividades que puedan resultar peligrosas para mi hijo & .85 & $.690-.935$ & .875 & $.719-.950$ \\
\hline $\begin{array}{l}\text { 20. Cuando pienso en mi infancia (la aparto de mi pensamiento... creo } \\
\text { que fue estupenda) }\end{array}$ & .85 & $.690-.935$ & .85 & $.690-.935$ \\
\hline $\begin{array}{l}\text { 21. Cuando era pequeña (nadie se mostraba cariñoso... mis padres me } \\
\text { querían) }\end{array}$ & .875 & $.719-.950$ & .85 & $.690-.935$ \\
\hline 22. Procuro llevar una alimentación equilibrada & .775 & $.606-.885$ & .825 & $.661-.919$ \\
\hline 23. Toco mi vientre para sentir los movimientos de mi hijo & .9 & $.750-.964$ & .975 & $.852-.996$ \\
\hline $\begin{array}{l}\text { 24. He comentado con mi pareja los cambios que el bebé producirá en } \\
\text { nuestras vidas }\end{array}$ & .9 & $.750-.964$ & .95 & $.816-.988$ \\
\hline $\begin{array}{l}\text { 25. Acaricio mi abdomen para que mi hijo sienta el cariño que le tengo o } \\
\text { para calmarlo }\end{array}$ & .875 & $.719-.950$ & .95 & $.816-.988$ \\
\hline 26. Voy a clases de preparación al parto & .9 & $.750-.964$ & .9 & $.750-.964$ \\
\hline 27. Le hablo a mi hijo, le pongo música, le leo cosas en voz alta. & .95 & $.816-.988$ & .975 & $.852-.996$ \\
\hline $\begin{array}{l}\text { 28. Me considero (poco agradable a los demás... del montón... alguien que } \\
\text { se hace querer) }\end{array}$ & .85 & $.690-.935$ & .9 & $.750-.964$ \\
\hline 29. Las relaciones con mi pareja (no existen... son malas... son excelentes) & .875 & $.719-.950$ & .9 & $.750-.964$ \\
\hline $\begin{array}{l}\text { 30. Encuentro que mi cuerpo actualmente (es horrible... tiene gracia... me } \\
\text { favorece) }\end{array}$ & .825 & $.661-.919$ & .875 & $.719-.950$ \\
\hline
\end{tabular}


En la segunda evaluación se obtuvieron mejores resultados, como se aprecia en la presente tabla solo once ítems no superaron el valor de .70 (Charter, 2003) en el límite inferior del IC al 95\%. Seis de los ítems observados por los jueces mejoraron luego de redefinirlos a partir de sus apreciaciones.

Se presentan los ítems que fueron observados, respecto a su relevancia, en el proceso de validación de contenido a través del juicio de expertos. Esta primera fuente de validez mostró que algunos ítems presentan una baja capacidad para medir la dimensión a la cual pertenece.

Tabla 2

Ítems observados en el proceso de validación de co+ntenido

\begin{tabular}{ll}
\hline \multicolumn{1}{c}{ Dimensión } & \multicolumn{1}{c}{ Ítems observados } \\
\hline $\begin{array}{l}\text { F1. Aproximación afectiva al feto y al } \\
\text { bebé a través del pensamiento. }\end{array}$ & $\begin{array}{l}\text { 12. Imagino cómo será mi bebé. } \\
\text { 22. He recogido información sobre el feto, sobre el bebé o sobre ambos. }\end{array}$ \\
$\begin{array}{l}\text { F2. aceptación del embarazo y } \\
\text { adaptación a la maternidad. }\end{array}$ & 21. Cuando se confirmó mi embarazo (no lo deseaba... lo acepté bien...) \\
$\begin{array}{l}\text { F3: aproximación afectiva a través de la } \\
\text { diferenciación del feto. }\end{array}$ & 20. He empezado a preparar algunas cosas para el nacimiento del bebé. \\
$\begin{array}{l}\text { F4. experiencias infantiles y cuidado } \\
\text { prenatal. }\end{array}$ & $\begin{array}{l}\text { 18. Cuando era pequeña (nadie se mostraba cariñoso... mis padres me } \\
\text { querían). }\end{array}$ \\
$\begin{array}{ll}\text { F5. aproximación afectiva a través de la } \\
\text { interacción con el feto. }\end{array}$ & $\begin{array}{l}\text { 3. Toco mi vientre para sentir los movimientos de mi hijo. } \\
\text { 17. Le hablo a mi hijo, le pongo música, le leo cosas en voz alta. }\end{array}$ \\
$\begin{array}{ll}\text { F6. aproximación afectiva a través del } \\
\text { bienestar emocional. }\end{array}$ & $\begin{array}{l}\text { 9. Las relaciones con mi pareja (no existen... son malas... son excelentes). } \\
\text { 11. Encuentro que mi cuerpo actualmente (es horrible... tiene gracia... me } \\
\text { favorece). }\end{array}$ \\
\hline
\end{tabular}

Por otro lado, en cuanto a la validez interna de la escala, se realizó el análisis factorial exploratorio para ello se utilizó el programa FACTOR 10.8.02 (LorenzoSeva \& Ferrando, 2007). El propósito del análisis fue explorar la estructura interna del instrumento; es decir, su dimensionalidad.

Respecto a la pertinencia del análisis factorial de los datos, el análisis factorial exploratorio indicó que la matriz de la determinante fue de 0.000034606622076 , la prueba de adecuación muestral a través del coeficiente Kaiser-Meyer-Olkin (KMO) obtuvo un valor de 0.88622 el cuál es considerado como bueno (Kaiser, 1970). Asimismo, la prueba de esfericidad de Bartlett (Bartlett, 1950) fue significativa $(\chi 2=3059.2 ; \mathrm{gl}=465 ; \mathrm{p}<0,001)$; estos resultados señalaron la posibilidad de realizar un análisis factorial (Kaplan y Saccuzzo, 2006).

Respecto a la agrupación que ofrece el AFE sugirió la existencia de 2 dimensiones, las cuales corresponderían a las variables latentes de medición; por un lado, la adaptación al embarazo y la vinculación afectiva prenatal. Además, el 
análisis nos ofreció que ítems estarían conformando cada factor. Estos resultados serán contrastados en el Análisis factorial confirmatorio (AFC).

Continuando con el proceso de obtención de evidencias sobre la validez interna del instrumento se obtuvieron las estimaciones de los modelos para comparar sus índices de ajuste. Se efectuó el AFC con el programa AMOS 24.0. el cual se utiliza el Modelo de ecuaciones estructurales (SEM).

Los modelos a evaluar fueron: a) la propuesta teórica de la escala, b) el modelo ofrecido por el análisis factorial exploratorio y c) modelo del AFE omitiendo ítems para un mejor ajuste.

Los resultados obtenidos de los dos últimos modelos aportaron resultados óptimos en los índices estimados en comparación con el modelo teórico propuesto por la autora.

Tabla 3

Comparación de los indices de ajuste estadístico de tres modelos de la Escala de adaptación y vinculación afectiva prenatal $(N=310)$.

\begin{tabular}{|c|c|c|c|c|c|c|}
\hline Modelos & $\chi^{2}$ & $\chi^{2 / g l}$ & SRMR & CFI & AIC & RMSEA \\
\hline Modelo 1 (propuesta teórica) & 941.13 & 2.335 & - & 0.801 & 1127.13 & $\begin{array}{c}0.066 \\
{[0.06-0.071]}\end{array}$ \\
\hline Modelo 2 (AFE- 31 ítems) & 1060.681 & 2.455 & .0665 & 0.768 & 1188.681 & $\begin{array}{c}0.069 \\
{[0.063-0.074]}\end{array}$ \\
\hline Modelo 3 (AFE- 21 ítems) & 245.833 & 1.308 & .0462 & 0.956 & 331.833 & $\begin{array}{c}0.032 \\
{[0.019-0.042]}\end{array}$ \\
\hline
\end{tabular}

Nota: $\mathrm{X} 2=$ Chi cuadrado, $\mathrm{gl}=$ grados de libertad, $\mathrm{SRMR}=$ raíz residual estandarizada cuadrática media, $\mathrm{RMSEA}=$ error cuadrático medio de aproximación, $\mathrm{CFI}=$ índice de ajuste comparativo, $\mathrm{AIC}=$ criterio de Akaiken, $\mathrm{IC}=$ intervalos de confianza

El modelo final compuesto por 21 ítems mostró mejores bondades de ajuste. Respecto a la razón entre $\chi 2$ y el número de grados de libertad $(\chi 2 / \mathrm{gl})$ se encontró un valor de 1.308 , de acuerdo con este valor debería ser menor a 2 (Tabachnick \& Fidell, 2001); no obstante, los modelos en comparación ofrecen valores superiores a este valor. En cuanto al Standardized Root Mean Square Residual (SRMR), Hu y Bentler (1998) sugieren un valor inferior a .08 el cual cumplen los tres modelos; nos obstante, el menor valor de este índice se encontró en el modelo 3 (SRMR= .0462). Para Hu y Bentler (1998) el RMSEA debe alcanzar un valor por debajo de 0.06 , por tanto, el único que cumple con dicho requerimiento fue el modelo 3 (RMSEA=.032) con un intervalo de confianza al 95\% (0.019-0.042). Asimismo, el índice de ajuste incremental CFI mostró un valor próximo a lo sugerido por Hu \& Bentler (1998) es decir, un valor mayor a .95 (CFI= .956). Finalmente, el índice de ajuste de parsimonia de Aiken, el modelo 3 mostró un valor menor a los hallados en los otros modelos 1 y $2(\mathrm{AIC}=331.833)$. 
Es importante señalar que el modelo 3 se obtuvo a partir del modelo ofrecido por el AFE; para ello, se eliminó consecutivamente, partir de las recomendaciones ofrecidas por el Índice de modificación del programa, aquellos ítems que no favorecían al modelo. Durante el proceso se eliminaron 10 ítems, el modelo final compuesto por 21 enunciados presenta los mejores valores.

A partir de los resultados sobre la dimensionalidad la escala, lo cuales sugirieron un modelo de dos factores, se calcularon los coeficientes de confiabilidad para cada una de las dimensiones. La confiabilidad fue estimación mediante el coeficiente omega (McDonald, 1999) el cual difiere del coeficiente alfa de Cronbach en el uso de las cargas factoriales de los ítems (Gerbing, \& Anderson, 1988), por lo tanto, los coeficientes obtenidos son más estables y reflejan un mejor nivel de fiabilidad (Timmerman, 2005). Además, un detalle relevante en el uso del coeficiente omega es que su cálculo no depende del número de ítems (McDonald, 1999).

En cuanto a los coeficientes de confiabilidad, la sub escalas Adaptación al embarazo y Vinculación afectiva prenatal obtuvieron valores de .746 y .749 respectivamente. Todos los coeficientes presentaron valores mayores a .70 por lo que fueron considerados aceptables (Campo-Arias. \& Oviedo, 2008).

\section{DISCUSIÓN}

El presente estudio brindó evidencias en cuanto a la validez de la escala y la propuesta teórica que la sustenta. Al respecto se encontró que los seis factores que conformaban la escala (Lafuente, 2008) representan aspectos vinculados estrechamente con las variables latentes, la adaptación al embarazo y la vinculación afectiva prenatal. Los hallazgos del estudio concluyeron que las seis dimensiones propuesta por Lafuente representan microdimensiones de los constructos adaptación y vinculación afectiva prenatal; por tanto, no se requiere su subdivisión, siendo necesaria una estructura solo con dos dimensiones.

Por otro lado, los hallazgos empíricos corroboran la propuesta de valorar conjuntamente la adaptación y la vinculación prenatal ya que ambas se encuentran estrechamente relacionadas. La correlación entre ambas variables, en el modelo final de 21 ítems, indican un valor de 80 .

\section{CONCLUSIONES}

- La evidencia empírica sugiere que no se requiere subdividir las variables adaptación y vinculación afectiva prenatal en dimensiones menores ya que están representan características de ellas.

- En cuanto a las evidencias de la validez de constructo de la escala, la estructura de dos dimensiones conformada por 21 ítems presenta 
mejores bondades de ajuste en comparación con la propuesta teórica y la estructura resultante del AFE sin omisión de ítems.

- Los hallazgos en cuanto a la confiabilidad de la escala indican valores adecuados para cada una de sus subescalas.

- La escala permite valorar oportunamente el estado actual de la vinculación afectiva prenatal, así como la adaptación al embarazo. Estos datos resultan imprescindibles para la elaboración de actividades de intervención en la población donde se identifiquen desajustes emocionales.

- Una escala con adecuadas propiedades psicométricas permite detectar tempranamente actitudes y conductas adaptativas frente al embarazo, así como aquellos comportamientos de rechazo y/o conductas negligentes de las gestantes. Un acompañamiento psicológico durante el periodo del embarazo y posterior a este contribuirá al desarrollo de un apego seguro en la diada madre/padre- hijo. 


\section{ANEXO A.}

ESCALA PARA LA EVALUACIÓN DE LA VINCULACIÓN AFECTIVA Y LA ADAPTACIÓN PRENATAL (EVAP)

(Versión adaptada por el servicio de psicología del INMP, 2018)

\section{SUB ESCALA- VINCULACIÓN AFECTIVA PRENATAL}

1. Imagino como es ahora mi hijo/a
a. No, me resulta imposible
b. No, no lo he intentado
c. Me cuesta imaginarlo.
d. Lo hago algunas veces
e. Lo hago con frecuencia.

2. Acaricio mi abdomen para que mi hijo/a sienta el cariño que le tengo y para calmarlo si está inquieto moviéndose demasiado:
a. No, porque no creo que vaya a darse cuenta.
b. No, porque me siento ridícula haciendo eso.
c. Casi nunca lo hago.
d. Sí, algunas veces.
e. Sí, con relativa frecuencia.

3. Imagino como será mi bebé en el futuro (su aspecto, su forma de ser, etc.)
a. No, me resulta imposible.
b. No, no le intentado
c. Me asusta hacerlo.
d. Lo hago algunas veces.
e. Lo hago con frecuencia y me resulta fácil.

4. Cuando mi hijo se mueve:
a. Me molesta.
b. Me pone un poco nerviosa. 

c. Me resulta indiferente.
d. Me llama la atención.
e. Me gusta y me emociona.

5. Le hablo a mi hijo/a, le canto, le pongo música y/o le leo cosas en voz alta:
a. No, porque creo que es completamente inútil.
b. No, porque no me sale de forma espontánea.
c. Sí, algunas veces.
d. Sí, cada tarde.
e. Sí, con frecuencia.

6. Puedo hacerme una cierta idea del carácter de mi hijo7a, por su forma de moverse dentro de mi (muy activo o muy reposado, por ejemplo).
a. No.
b. Casi nada.
c. Tengo dudas.
d. Un poco.
e. Sí.

7. He empezado a preparar algunas cosas para el nacimiento de mi bebé (su habitación, su ropa, la cuna, los biberones, etc.)
a. No, aún no he preparado nada porque no tengo muchas ganas, estoy poco motivada.
b. No, aún no he tenido tiempo.
c. He preparado pocas cosas ya tendré tiempo más adelante.
d. He preparado algunas cosas.
e. Ya lo he preparado casi todo.

8. Me he interesado en conocer más sobre la maternidad
a. No, nunca.
b. Algo, hablando con otras madres 

c. Algo, hablando con otras madres y leyendo algunas cosas.
d. Bastante (de otras madres, libros, revistas, etc.)
e. Mucha (de otras madres, libros, revistas, matronas, médicos, etc.).

9. He pensado en el regreso a casa desde el hospital con $\mathrm{m}$ i bebé en brazos
a. No, creo que no es importante pensar en esas cosas.
b. No, ¿para qué? Aún falta mucho
c. No, pero pensaré en ello más adelante
d. Sí, lo he pensado algunas veces.
e. Sí, pienso en ello con frecuencia.

\section{SUB ESCALA - ADAPTACIÓN AL EMBARAZO}

10. Cuando pienso en el futuro al lado de mi bebé:
a. Me siento mal.
b. Me angustia
c. Tengo muchas dudas y temores
d. Me considero una madre capaz.
e. Sé que le gustaré y lo haré bien.

11. Encuentro que mi cuerpo actualmente:
a. Ha cambiado y no es de mi agrado.
b. Ha perdido su forma.
c. Ha cambiado, pero no es un problema para mí.
d. Ha cambiado, pero intento verme bien.
e. Me agrada mi nuevo aspecto.

12. Evito realizar actividades que puedan resultar peligrosas para mi hijo/a (llevar excesivo peso, hacer ejercicios demasiados violentos, fatigarse en exceso, etc.)
a. No, eso de cuidarse de los peligros son tonterías.
b. No me preocupo demasiado de so 

c. Si, algunas veces.
d. Sí, siempre que puedo.
e. Sí, siempre.

13. Voy a clase de preparación de parto
a. No ni pienso hacerlo, no las considero útiles.
b. No, estoy demasiado ocupada.
c. Aun no, lo hare más adelante.
d. Sí, porque quiero que el embarazo y el parto salga bien.
e. Sí, porque quiero estar bien preparada para el nacimiento de mi hijo/a.

14. Durante mi infancia y adolescencia:
a. Mi padre me maltrataba bastante, porque me lo merecía.
b. Mi padre no se portó bien conmigo.
c. Mi padre no era demasiado cariñoso conmigo
d. Mi padre era bueno conmigo, pero no me entendía bien.
e. Mi padre me quería y supo educarme bien.

15. Durante mi infancia y adolescencia:
a. Mi madre me maltrataba bastante, porque me lo merecía.
b. Mi madre no se portó bien conmigo.
c. Mi madre no era demasiado cariñosa conmigo
d. Mi madre era buena conmigo, pero no me entendía bien.
e. Mi madre me quería y supo educarme bien.

16. Cuando se confirmó mi embarazo:
a. No quería creerlo, la verdad es que aún no deseo tener este hijo.
b. Me costó mucho hacerme la idea y no sé aun si lo he aceptado del todo.
c. Me costó algún tiempo aceptarlo, pero ya lo tengo bien asumido. 
d. Lo acepté bien porque aunque yo no lo había planeado, quería quedar embarazada.

e. Me puse contentísima, ya que era todo lo que quería.

17. Creo que el embarazo:
a. Es un auténtico fastidio.
b. Es algo por lo que no hay más remedio que parar si quieres tener un hijo/a.
c. Es una época como cualquier otra.
d. Es algo nuevo interesante, aunque pueda ocasionar algunas molestias.
e. Es algo muy especial, es un privilegio ser mujer en este caso.

18. Me resulta fácil imaginarme a mí misma dando de comer al bebé, cuidándolo, cambiándole los pañales, durmiéndolo, etc.

a. Para mi es imposible imaginar todo eso.

b. Me cuesta mucho esfuerzo imaginarlo.

c. Me lo imagino a veces, pero no sé si sabré hacerlo.

d. Me lo imagino bastantes veces.

e. Me lo imagino muchas veces y con facilidad.

19. Mi pareja y yo hablamos de nuestro futuro bebé:
a. Nunca
b. Casi nunca
c. Algunas veces
d. Bastante.
e. Mucho.

20. Siempre me han gustado los niños
a. No, ahora siguen sin gustarme.
b. No, pero los tolero.
c. Ni me gustan, ni me disgustan. 
d. Sí, pero me cuesta ganármelos.

e. Sí, me lo paso bien con ellos y suelo tener una buena relación.

21. El embarazo

a. Me ha producido muchas molestias (náuseas, vómitos, dolores) y no quiero volver a quedar embarazada

b. Me ha producido algunas molestias, que me han tenido bastante preocupada.

c. Me ha producido algunas molestias, pero eso es bastante común y sé que van a pasar.

d. Apenas me ha producido molestias, creo he tenido suerte.

e. Ha sido excelente, no he sentido ninguna molestia.

\section{REFERENCIAS}

American Educational Research Association, American Psychological Association, \& National Council on Measurement in Education (2014). Standards for educational and psychological testing. Washington, DC: American Educational Research Association.

Aiken, L. R. (1985). Three coefficients for analyzing the reliability and validity of ratings. Educational and Psychological Measurement, 45, 131-142.

Aiken, L. R. (1996). Test psicológicos y evaluación. México, D.F.: Prentice Hall

Ato, M.; López, J. \& Benavente, A. (2013). Un sistema de clasificación de los diseños de investigación en psicología. Anales de psicología, 29(3), 1038-1059

Campo-Arias,A. \& Oviedo, H. C. (2008). Propiedades psicométricas de una escala: la consistencia interna. Rev Salud Publica, 10(5), 831-839.

Comrey, A. L. y Lee, H. B. (1992). A first course in factor analysis. Hillsdale, NJ: Erlbaum

Charter, R. A. (2003). A breakdown of realiability coefficients by test type and reliabilily method, and the clinical implication of low reliability. Journal of General Psycholigyst, 130, (3), 290- 304.

Gerbing, D. W. \& Anderson J. C. (1988). An update paradigm for scale development incorporating unidimentionality and its assessment. Journal of Marketing Research, 25 (2), pp. 186-192

Hidalgo MV y Menéndez S. (2003). La pareja ante la llegada de los hijos e hijas. Evolución de la relación conyugal durante el proceso de convertirse en padres y madres. Infancia y Aprendizaje, 26 (4): 469-483 
Hughes C. (1987). Assessing the pregnant family. En L.N. Sherwen (Ed.). Psychosocial dimensions of the pregnant family. New York: Springer Pub. Co.

Hu, L. \& Bentler, P. (1998). Fit indices in covariance structure modeling: Sensivity to underparameterized model misspecification. Psychological Methods, 3(4), 424453.

Kaiser, H. F. (1970). A second generation Little Jiffy. Psychometrika, 35(4), 401-415. https://doi.org/10.1007/BF02291817

Kane, M. T. (2009). Validating the interpretations and uses of test scores. En R. W. Lissitz (Ed.), The concept of validity (pp. 39-64). Charlotte, NC: Information Age Publishing.

Kaplan, R. M., \& Saccuzzo, D. P. (2006). Pruebas psi-cológicas. Principios, aplicaciones y temas. México: Thomson.

Lederman, R. \& Weis, K. (2009). Psychosocial adaptation to pregnancy: Seven dimensions of maternal development. Third Edition. New York. Springer.

Lafuente, Ma (2008). La escala EVAP (Evaluación de la Vinculación Afectiva y la Adaptación Prenatal). Un estudio piloto. Index de Enfermería, 17 (2), p.133-137

Lorenzo-Seva, U., \& Ferrando, P. (2007). FACTOR: A computer program to fit the exploratory factor analysis model. University Rovira y Virgili

McDonald, R. P. (1999). Test theory: A unified treatment. Mahwah: Lawrence Erlbaum Associates, Inc.

Merino, C. \& Segovia, J. (2009). Intervalos de confianza asimétricos para el índice la validez de contenido: Un programa Visual Basic para la V de Aiken. Anales de psicología, 25, (1), 169-171

Montero, I. y León, O. (2002). Clasificación y descripción de las metodologías de investigación en Psicología. International Journal of Clinical and Health Psychology, 2(3), 503-508.

Oiberman, Alicia; Galíndez, Elena Psicología perinatal: Aplicaciones de un modelo de entrevista psicológica perinatal en el posparto inmediato Revista del Hospital Materno Infantil Ramón Sardá, 24(3), 2005, 100-109

Pedrosa, I., Suárez-Álvarez y García-Cueto, E. (2013). Evidencias sobre la Validez de Contenido: Avances Teóricos y Métodos para su Estimación [Content Validity Evidences: Theoretical Advances and Estimation Methods]. Acción Psicológica, 10(2),10-20. http://dx.doi.org/10.5944/ap.10.2.11820

Rodrigues, A-R, Niñirola- Molina, M.E y Martínez, M.D (1999). Atención integral durante el embarazo y postparto. Mente en blanco. Revista de psicología, 1, 29-31

Sireci S. \& Padilla J. (2014). Validating assessments: introduction to the special section. Psicothema, 26 (9), 7-9.

Tabachnick, B., \& Fidell, L. (2001). Using multivariate statistics (4th ed). Boston: Allyn $\&$ Bacon. 
Timmerman, M. (2005). Factor analysis. Recuperado de http://www.ppsw.rug. $\mathrm{nl} / \sim$ metimmer/FAMET.pdf

Ventura-León, J. (2018). Validez basada en la estructura interna: reanálisis al artículo de Dávila y Nevado, Educación médica 19(3), 131-190 